\title{
Chemical Bonding Analysis of AIN Polytypes by ELNES
}

T. Mizoguchi, M. Kunisu, M. Yoshiya*, I. Tanaka, H. Adachi, P. Rulis**, and W. Y. Ching**

Department of Materials Science and Engineering, Kyoto University, Sakyo, Kyoto 606-8501, Japan

* Materials Science Division, Lawrence Berkeley National Laboratory, 1 Cyclotron Road, LBNL, CA, 94720, USA

** Department of Physics, University of Missouri-Kansas City, Kansas City, MO, 64110-2499 USA

AlN is known to show three polytypes, wurtzite, zinc-blend, and rock salt structure. However, the ELNES from wurtzite have been only reported until now [1]. In this study, the first principles orthogonalized linear combination of atomic orbital (OLCAO) method $[2,3]$ has been made to predict and interpret the differences of ELNES among these polytypes.

The top of figure 1 shows the theoretical and experimental Al- $\mathrm{L}_{2,3}$ ELNES of wurtzite structure. The theoretical spectrum was obtained using the large supercell composed of 108 atoms. The theoretical transition energy was calculated by subtraction the total energy at the ground state from the core-hole state. It can be found that the theoretical spectrum can quantitatively reproduce the experimental spectrum. Al-L $\mathrm{L}_{2,3}$ ELNES of other phases were also shown on the same figure. Because only $\mathrm{Al}$ in rock-salt structure is coordinated 6 nitrogen, the spectral features are clearly different from other phases. In the case of zinc-blend structure, the main features of spectrum are almost same as that of wurtzite. But small difference can be predicted at the spectral threshold, peak A. The feature of peak $\mathrm{A}$ is broader than the wurtzite phase.

Figure 2 shows the $\mathrm{Si}-\mathrm{L}_{2,3}$ ELNES from wurtzite and zinc-blend $\mathrm{SiC}$. In the case of $\mathrm{SiC}$, experimental spectrum from zinc-blend structure was reported [4]. The experimental spectrum can be quantitatively reproduced by the theoretical spectrum using the large supercell composed of 128 atoms. The same differences as AlN can be shown at the spectral threshold.

Origins of these differences are discussed in detail.

\section{References}

[1] V. Serin, C. Colliex, R. Brydson, S. Matar, and F. Boucher, Phys. Rev. B, 58 (1998) 5106.

[2] W. Y. Ching, J. Am. Ceram. Soc., 73 (1990) 3135.

[3] S-D. Mo and W. Y. Ching, Phys. Rev. B, 62 (2000) 7901.

[4] K. Kaneko, M. Kawasaki, T. Nagano, N. Tamari, and S. Tsurekawa, Acta Mater., 48 (2000) 903. 


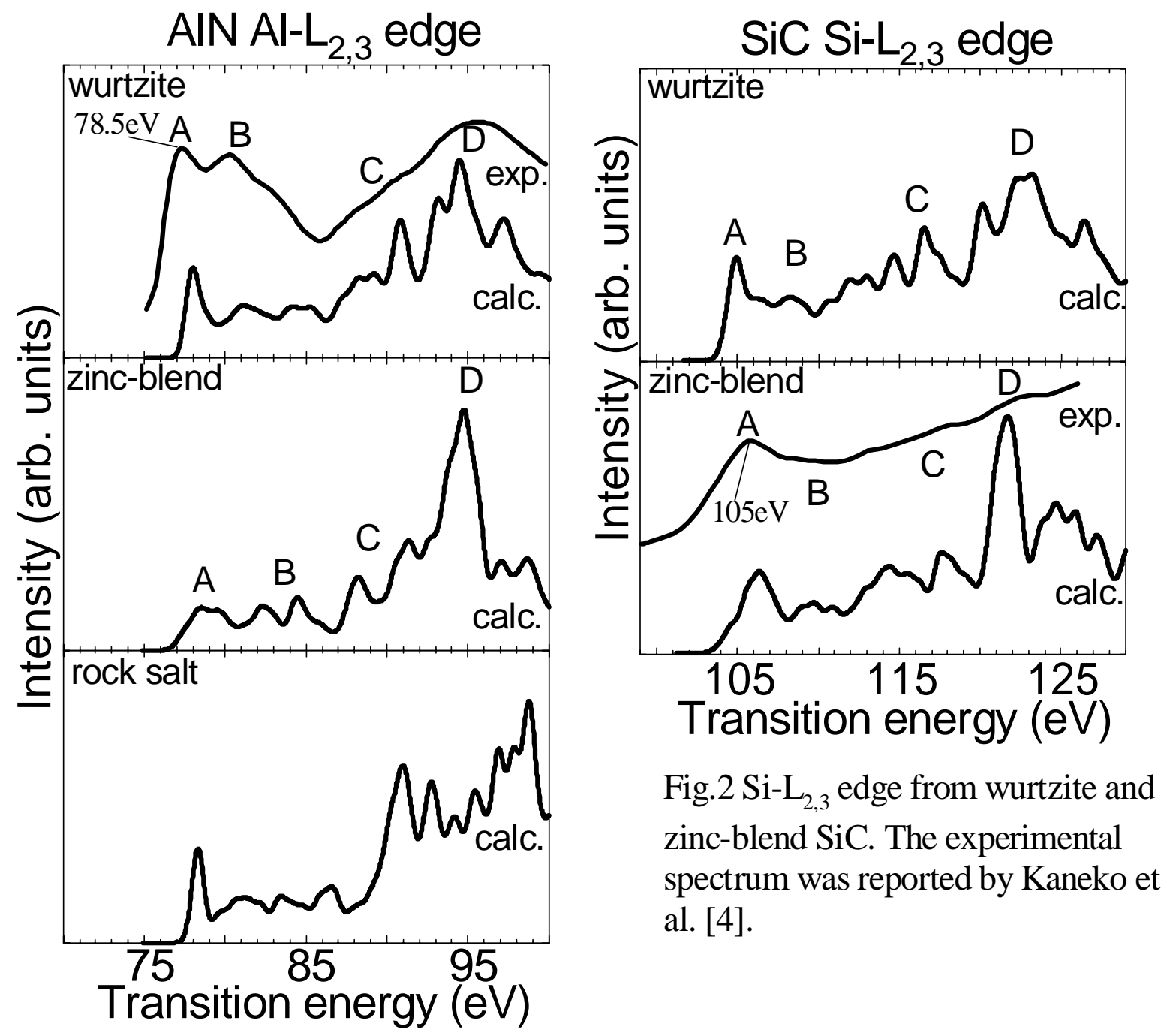

Fig.1 (top to bottom) Al- $\mathrm{L}_{2,3}$ edge from wurtzite, zinc-blend, and rock salt AlN. The experimental spectrum was reported by Serin et al. [1]. 